\title{
Broadband antireflection coatings for visible and infrared ranges
}

\author{
F. Lemarquis ${ }^{1}$, T. Begou ${ }^{1}$, A. Moreau ${ }^{1}$, F. Lemarchand ${ }^{1}$ and J. Lumeau1, ${ }^{1}{ }^{*}$
}

${ }^{1}$ Aix-Marseille Université, CNRS, Centrale Marseille, Institut Fresnel,

UMR 7249, 13013 Marseille, France

*julien.lumeau@fresnel.fr

\section{ICSO 2018}

International Conference on Space Optics

9-12 October 2018, Chania, Greece 


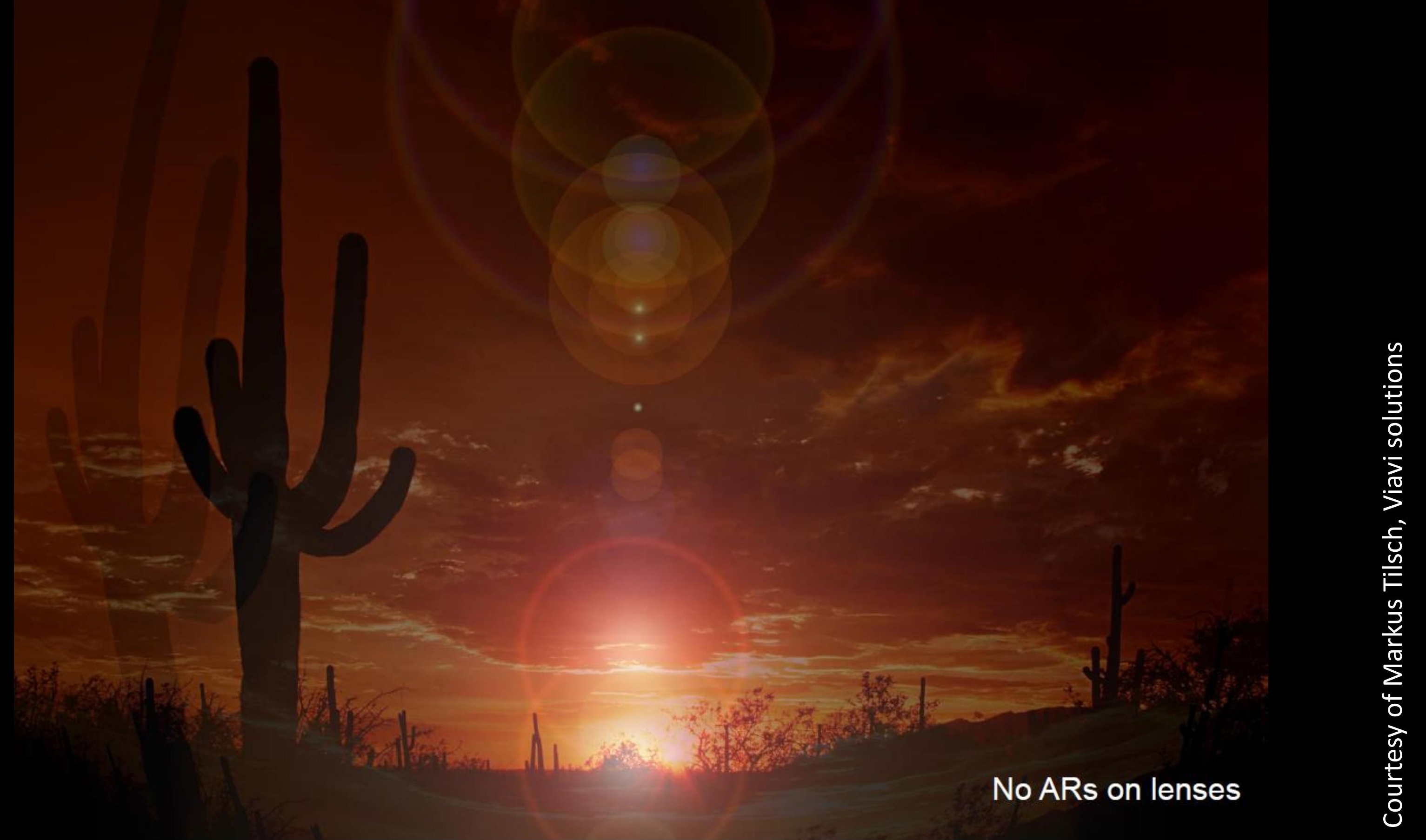




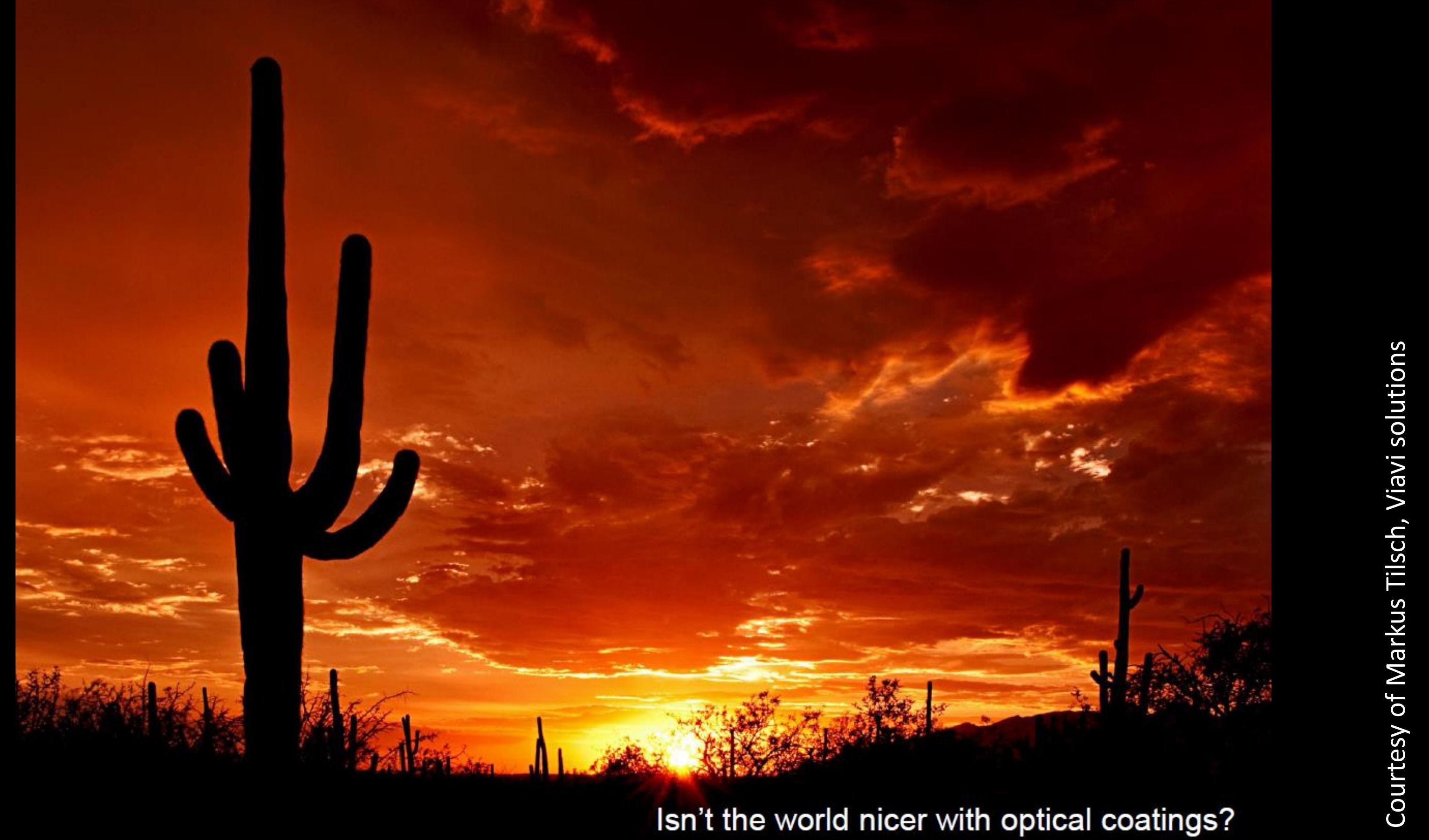


Antireflection coating

Single wavelength AR coating

- AR for glass at single wavelength (e.g. $600 \mathrm{~nm}$ ): $0.3 \mathrm{H} \mathrm{1.3L}$

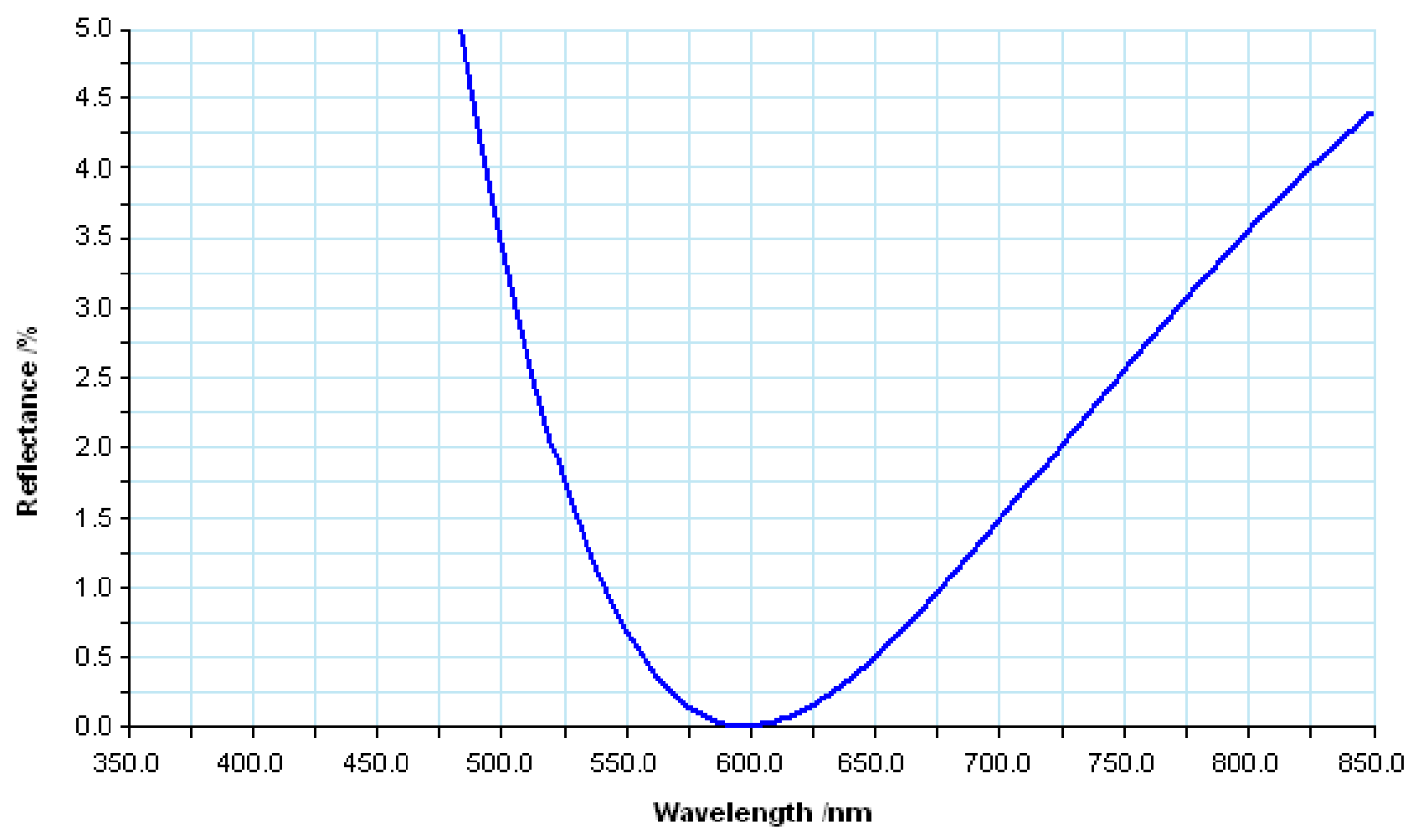




\section{Antireflection coating Broadband AR - Rules of thumb}

- Best ARC performances are achieved when using the highest and lowest possible refractive indices.

- The average residual reflection that can be achieved with multilayer structures depends on the required spectral range, the broader this range, the higher the residual reflection.

- The performances of antireflection treatments are indeed very sensitive to thickness variations of the layers. As a consequence, increasing the number of layers of a coating stack in order to theoretically improve its spectral performances might finally result in poorer performances than a simpler design. 
Antireflection coating

\section{Studied coatings}

- An antireflection coating for silica substrates optimized for normal incidence and covering the 400-1100 $\mathrm{nm}$ spectral range.

- An antireflection coating for silica substrates optimized for oblique incidence $\left(0\right.$ to $\left.45^{\circ}\right)$ and covering the $400-900 \mathrm{~nm}$ spectral range.

- An antireflection coating for ZnSe substrates optimized for normal incidence and covering the 1.5-15 $\mu \mathrm{m}$ spectral range. 


\section{Antireflection coating Order of magnitude}

- Substrate = silica

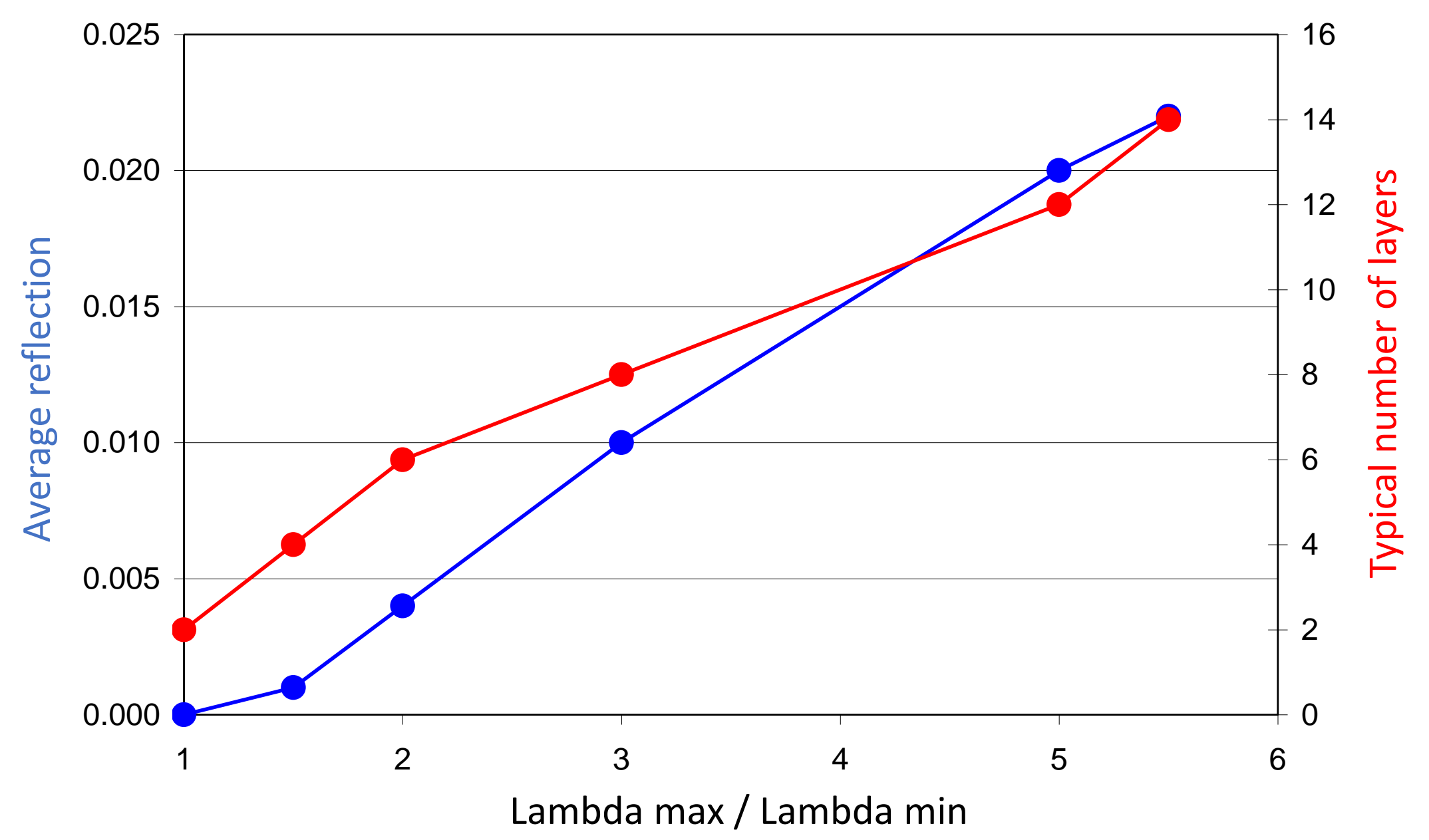




\section{0-1100 nm ARC at normal incidence}

Design $-\mathrm{Nb}_{2} \mathrm{O}_{5} / \mathrm{SiO}_{2}$

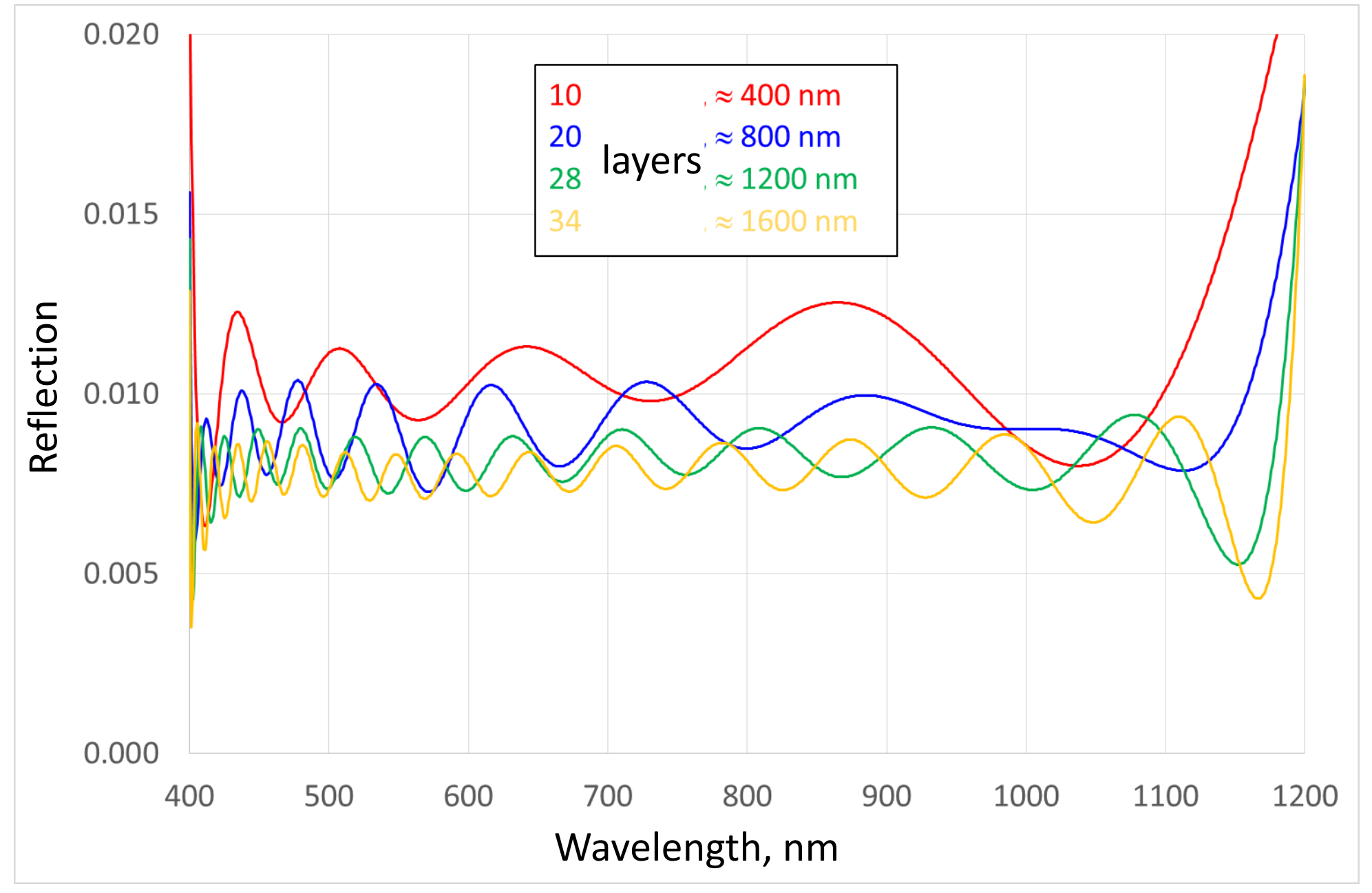

- Average reflection cannot be lower than $0.8 \%$

- Increasing the number of layers has a limited effect on average reflection

- Increasing the number of layers results in sharper change of reflection at boundaries 


\section{0-1100 nm ARC at normal incidence}

\section{Error sensitivity}

\section{0-layer design}

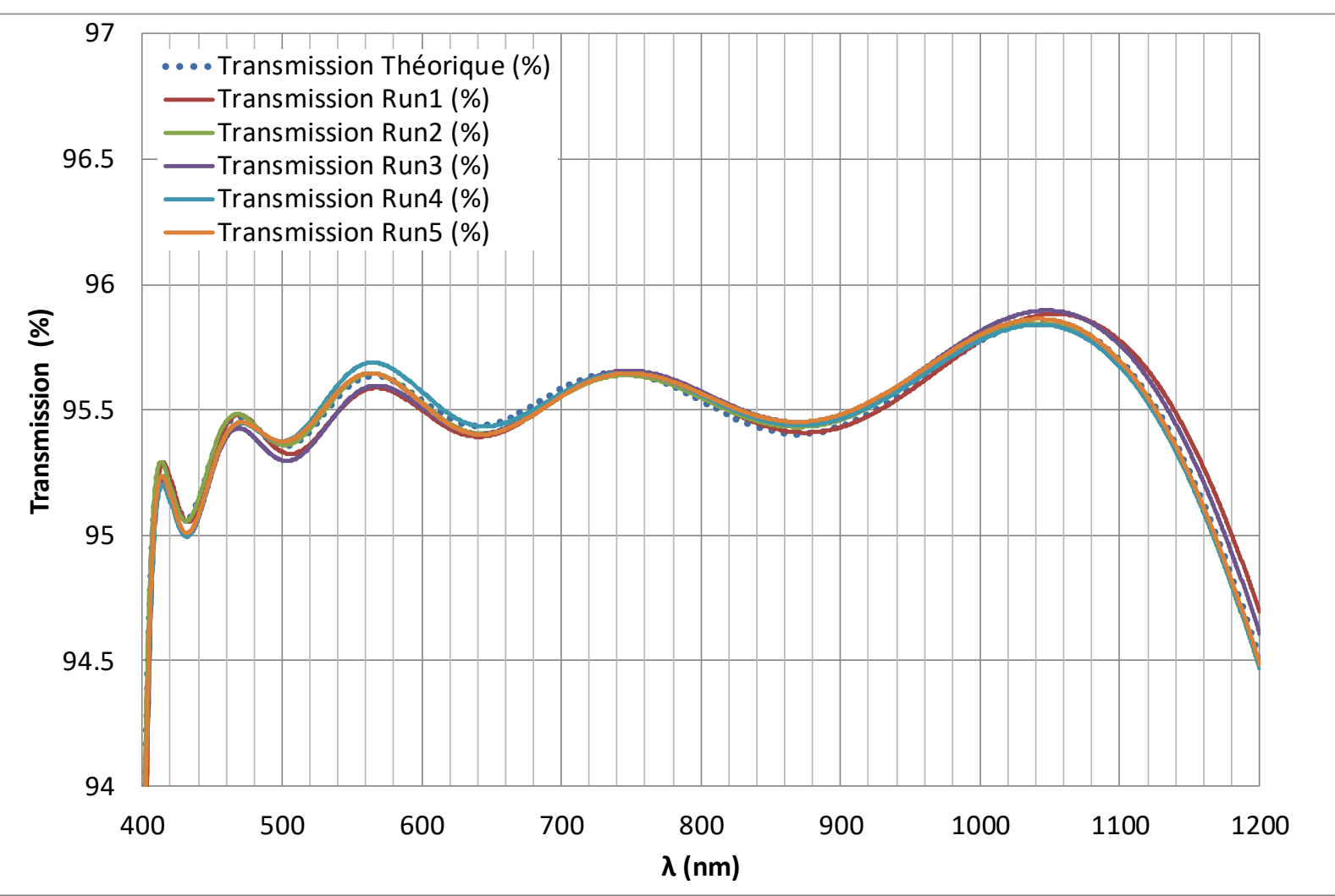

\section{4-layer design}

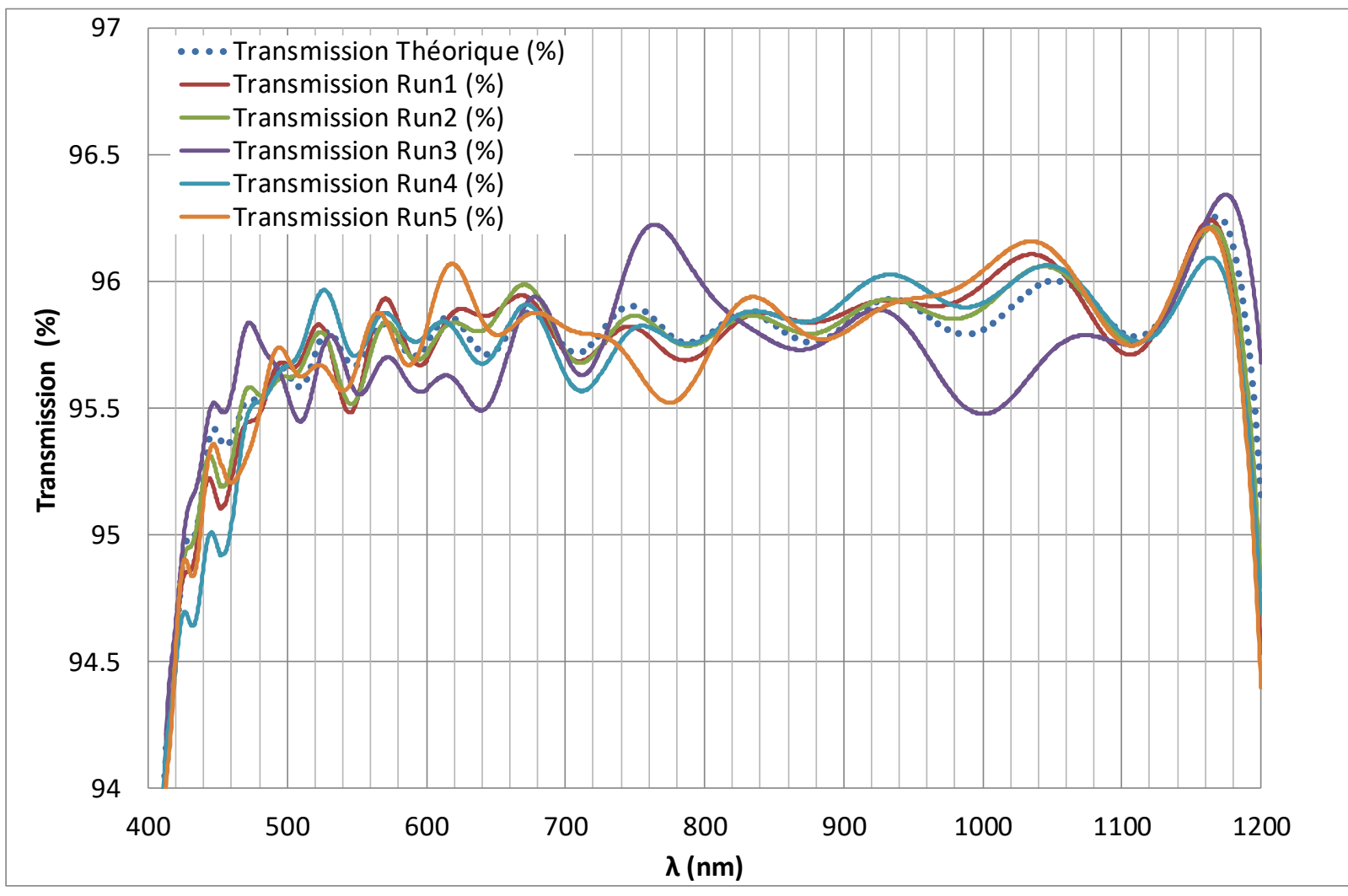




\section{0-1100 nm ARC at normal incidence}

Selected design

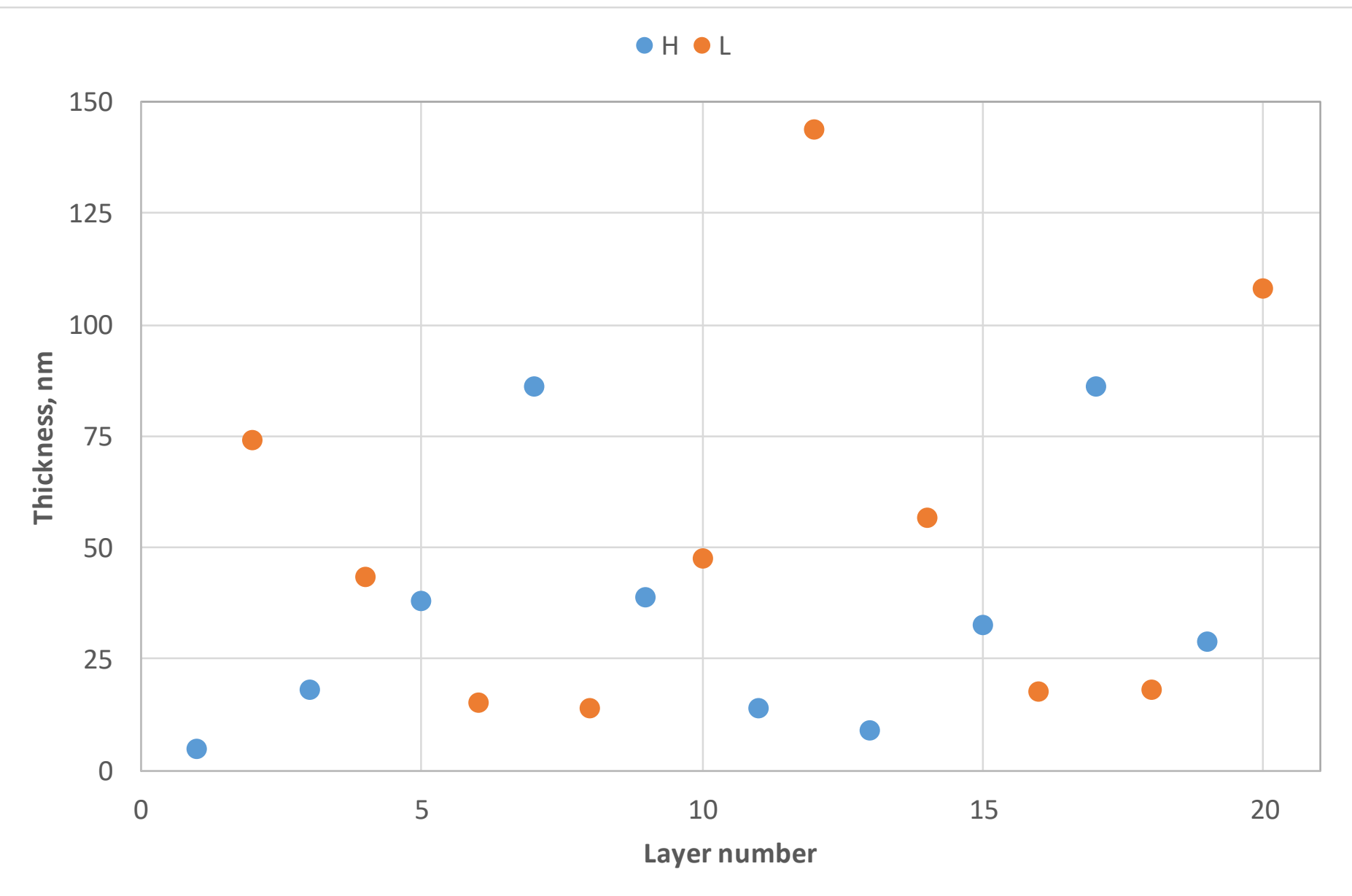

- Substrate = silica

- $\mathrm{H}=\mathrm{Nb}_{2} \mathrm{O}_{5}$

- $\mathrm{L}=\mathrm{SiO}_{2}$

- Number of layers $=20$

- Total thickness $=900 \mathrm{~nm}$

- Thinnest layer $=5 \mathrm{~nm}$

- Thicker layer $=144 \mathrm{~nm}$ 


\section{Deposition technology Bühler HELIOS machine}

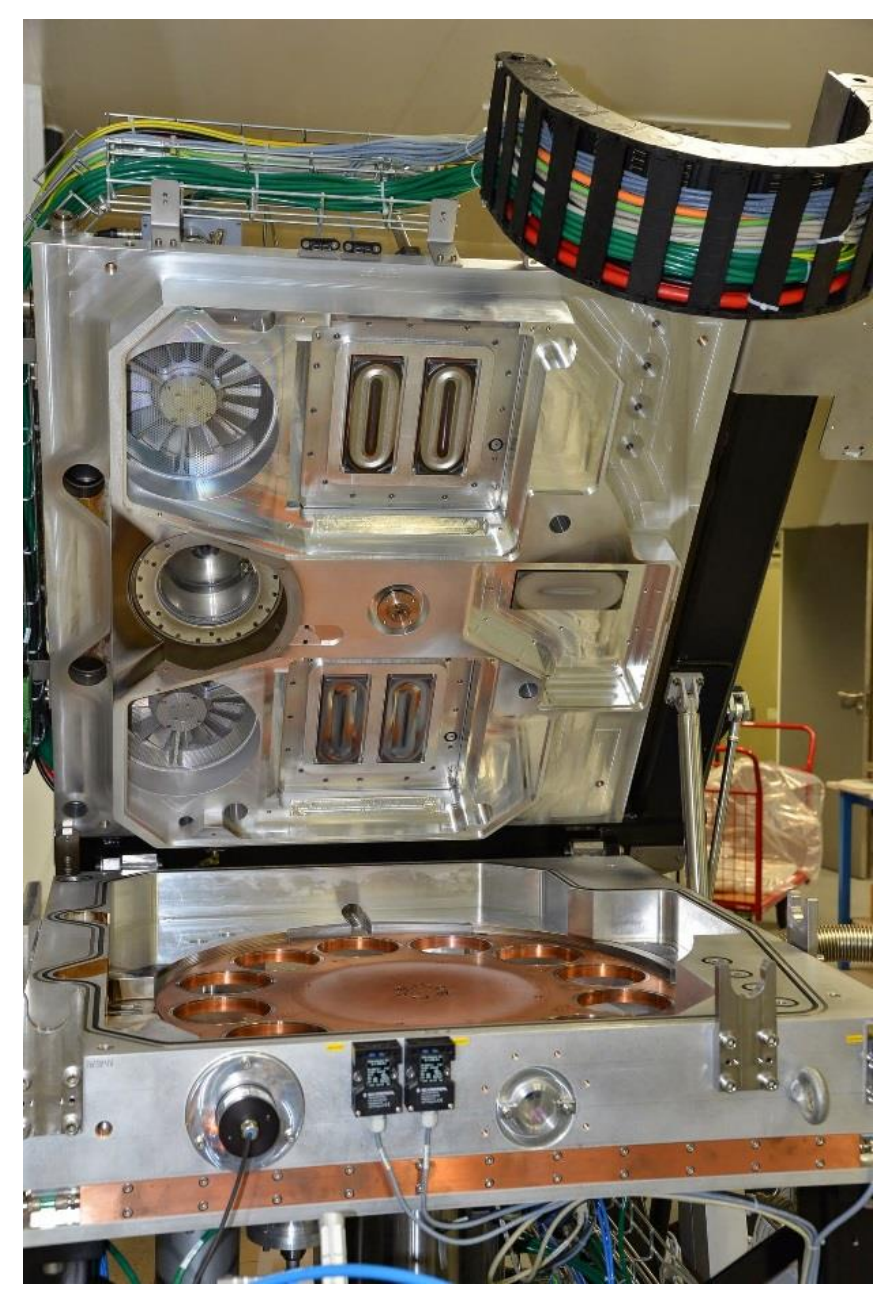

- 12 sample holders (1 dedicated to optical monitoring).

- Plate rotation at $240 \mathrm{rd}$.min-1.

- Load-lock.

- 4 process zones:

- 1 assistance plasma source,

- 2 MF magnetron sputtering sources (dielectric material deposition),

- 1 DC sputtering source (metallic material deposition).

- Real time monochromatic optical monitoring of the deposition in transmission.

- Available targets : $\mathrm{Si}, \mathrm{Nb}, \mathrm{Hf}, \mathrm{Ta}, \mathrm{Ag}, \mathrm{Cr}$.

- Available Gas: Ar, O2, N2. 


\section{0-1100 nm ARC at normal incidence \\ Experimental demonstration - single side}

Optical monitoring strategy:

- layers 1 to 8: optical monitoring at $410 \mathrm{~nm}$ on test glass 1 ,

- layers 9 to 20: optical monitoring at $470 \mathrm{~nm}$ on test glass 2 .
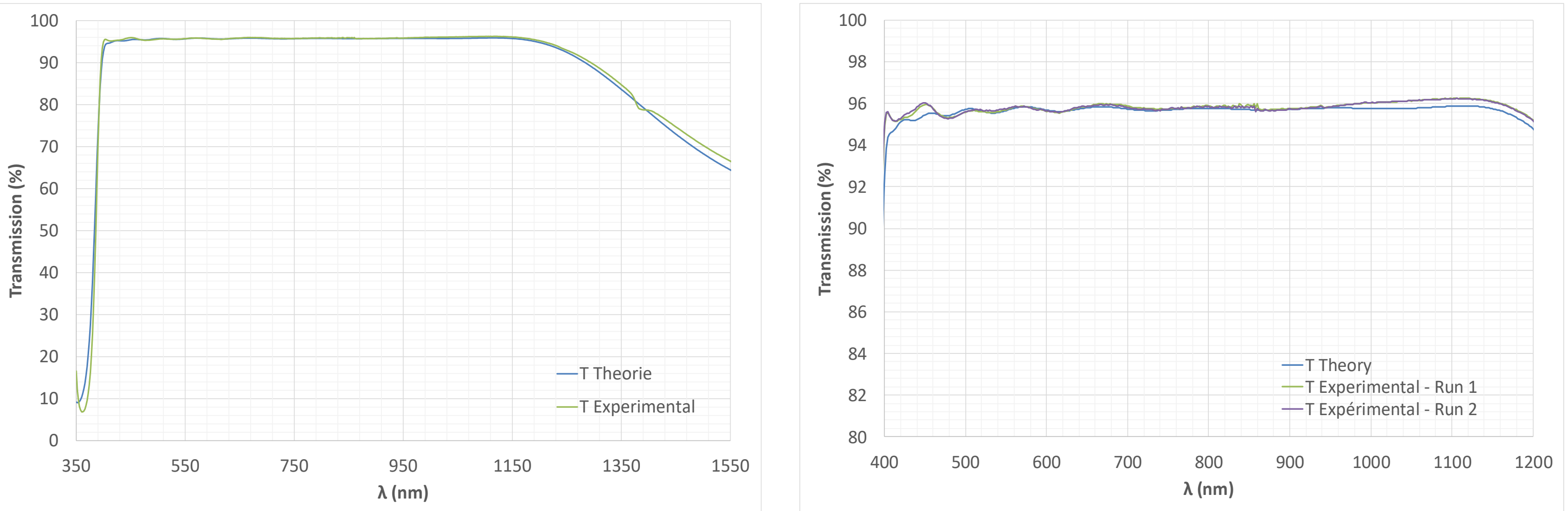


\section{0-1100 nm ARC at normal incidence}

Experimental demonstration - dual side

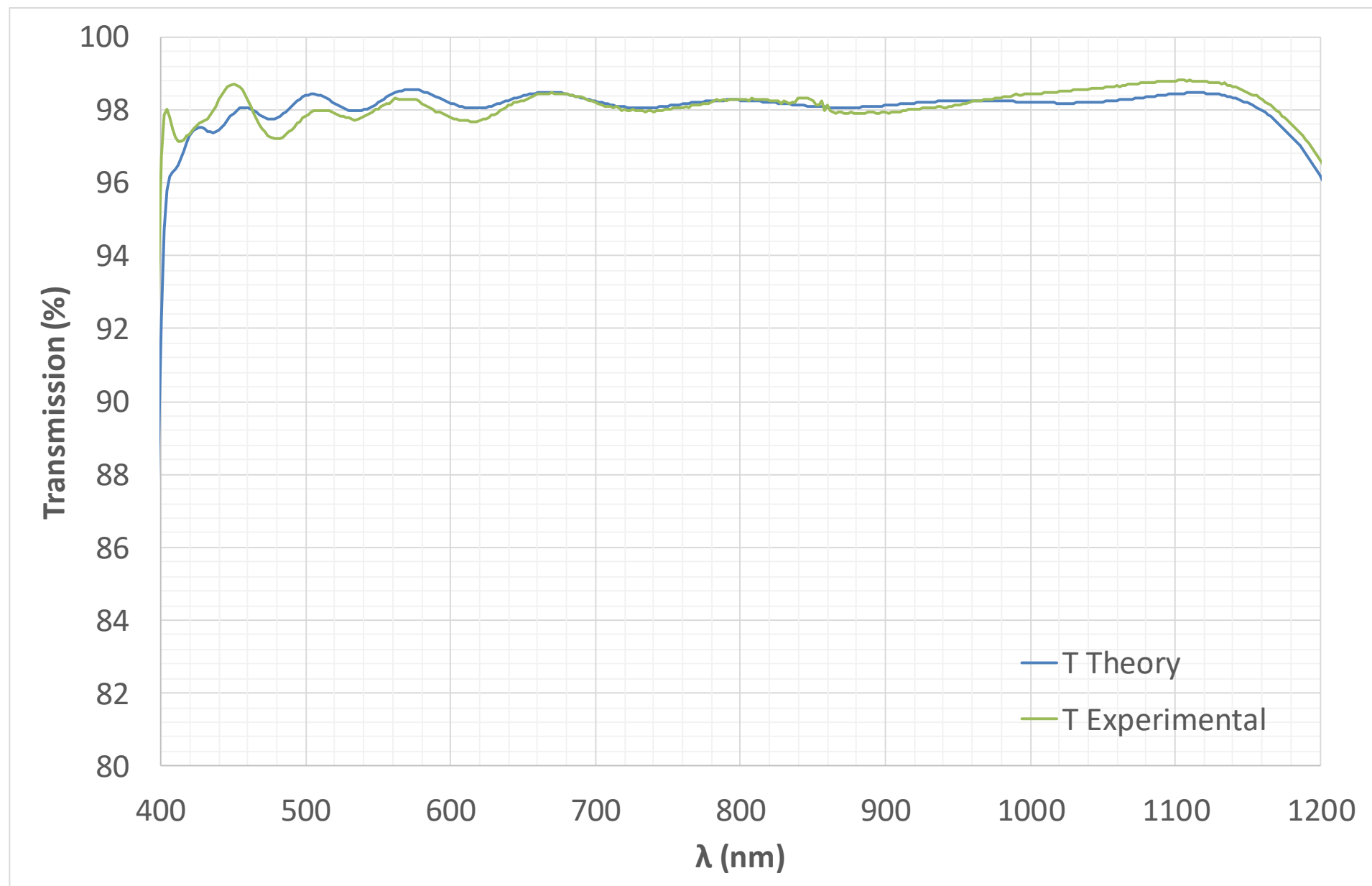




\section{0-900 nm ARC at oblique incidence Design $-\mathrm{Nb}_{2} \mathrm{O}_{5} / \mathrm{SiO}_{2}$}

Increasing the incidence results in a blue shift of the spectral profile and a split between $\mathbf{S}$ and $\mathbf{P}$ polarizations (in general $R_{S}>R_{P}$ ). These changes are larger and larger as soon as incidence is increased.
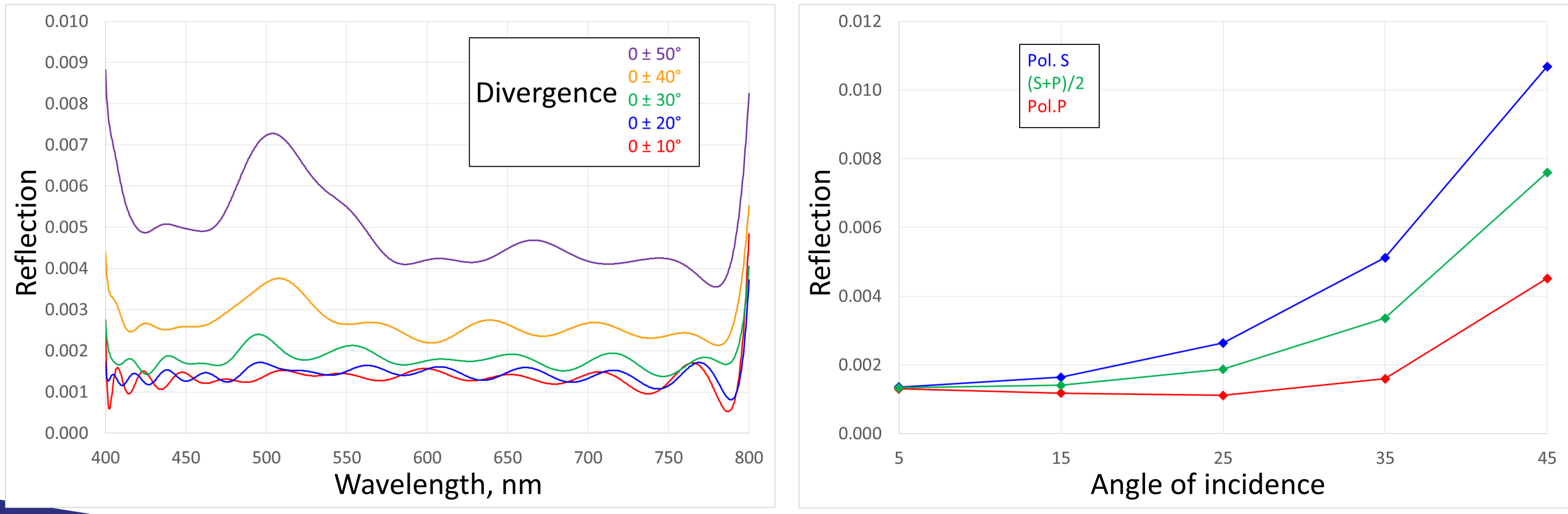


\section{0-900 nm ARC at oblique incidence}

Experimental

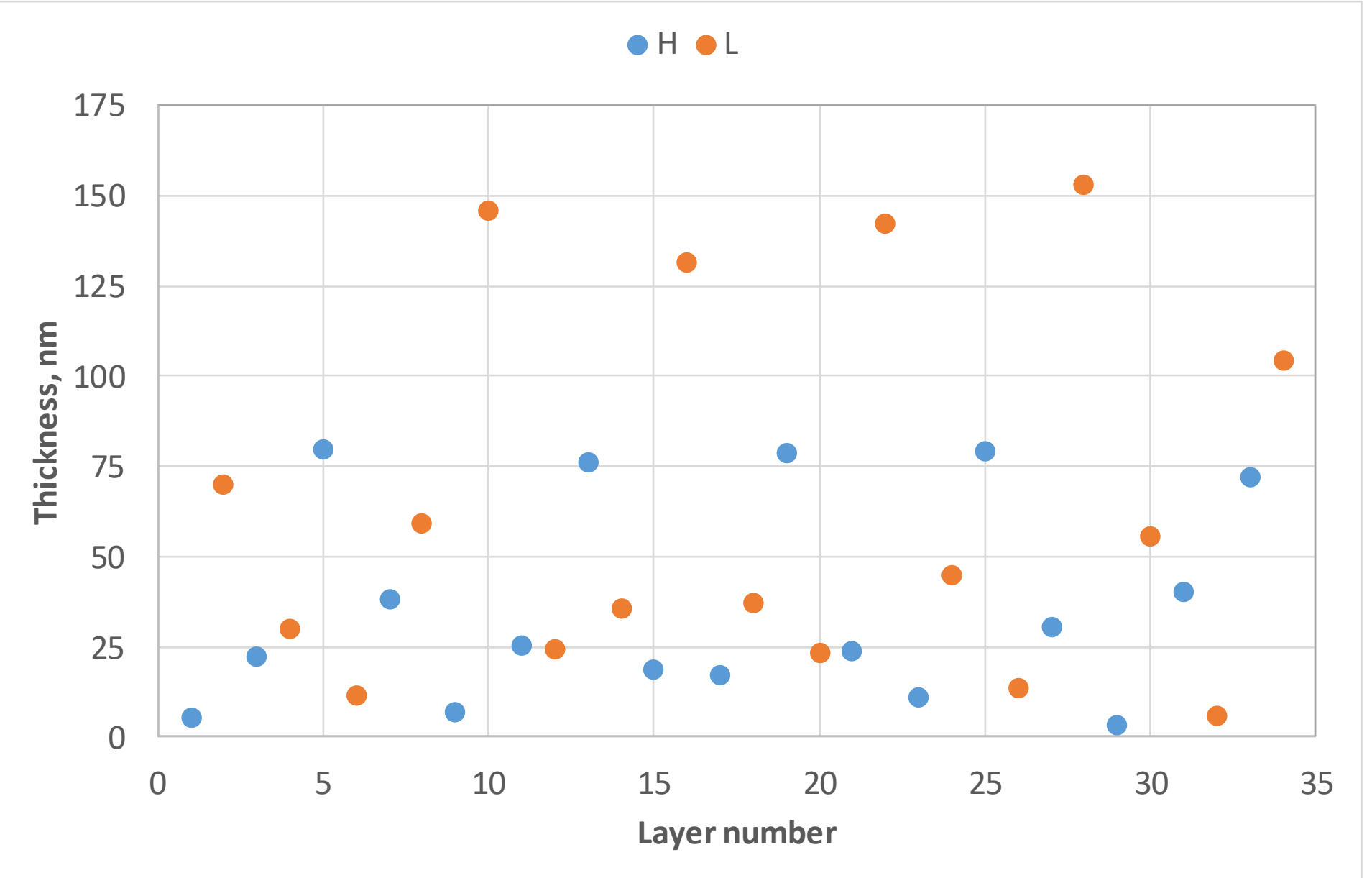

- Substrate = silica

- $\mathrm{H}=\mathrm{Nb}_{2} \mathrm{O}_{5}$

- $\mathrm{L}=\mathrm{SiO}_{2}$

- Number of layers $=34$

- Total thickness $=1700 \mathrm{~nm}$

- Thinnest layer $=3.2 \mathrm{~nm}$

- Thicker layer $=153 \mathrm{~nm}$ 


\section{0-900 nm ARC at oblique incidence}

\section{Experimental}

Optical monitoring strategy:

- layers 1 to 12: optical monitoring at $430 \mathrm{~nm}$ on test glass 1 ,

- layers 13 to 24: optical monitoring at $505 \mathrm{~nm}$ on test glass 2,

- layers 25 to 34 : optical monitoring at $450 \mathrm{~nm}$ on test glass 3 .
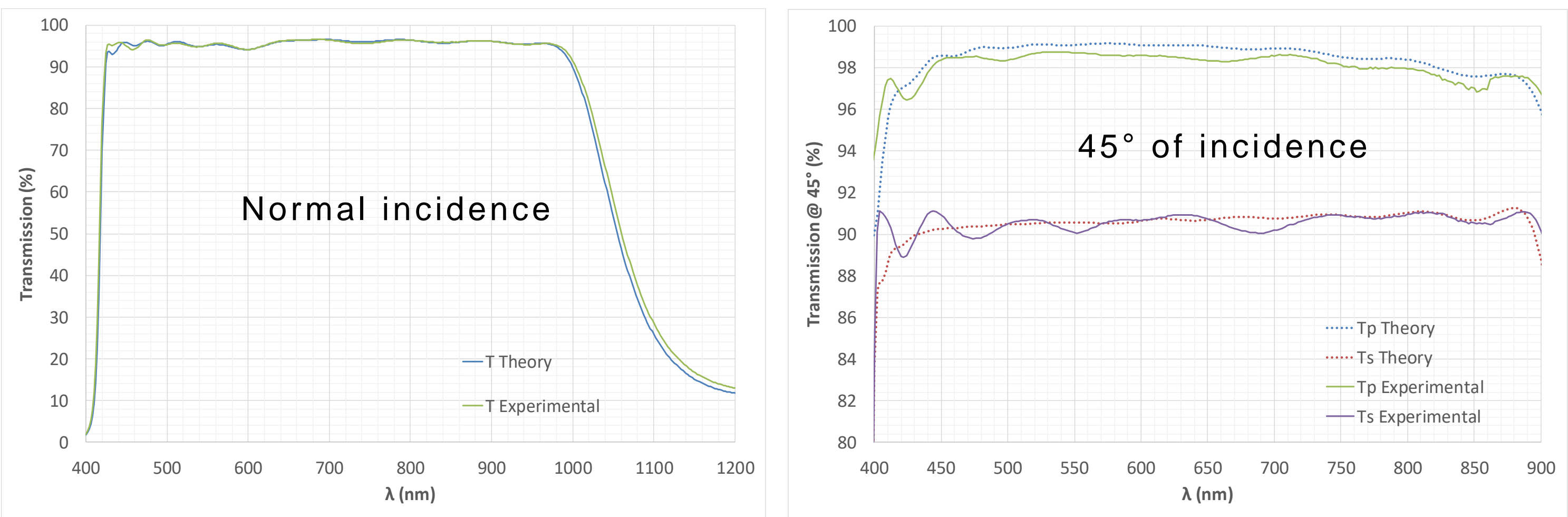


\section{0-900 nm ARC at oblique incidence}

\section{Experimental}

Optical monitoring strategy:

- layers 1 to 12: optical monitoring at $430 \mathrm{~nm}$ on test glass 1 ,

- layers 13 to 24: optical monitoring at $505 \mathrm{~nm}$ on test glass 2,

- layers 25 to 34 : optical monitoring at $450 \mathrm{~nm}$ on test glass 3 .
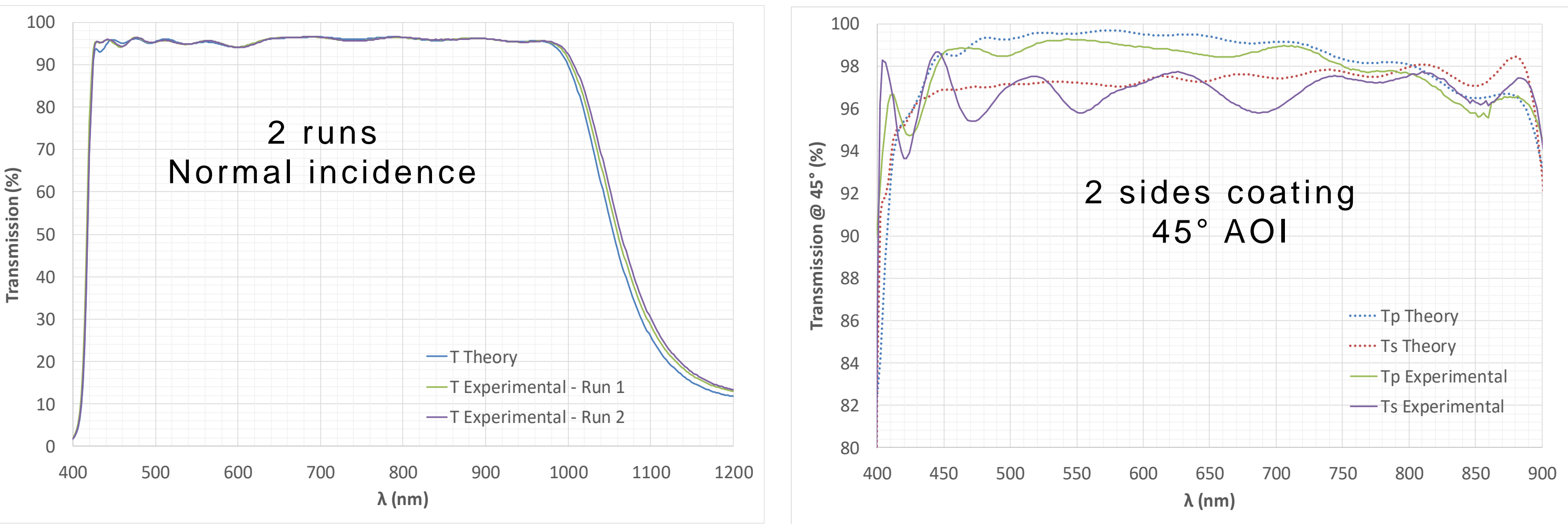


\section{5-15 $\mu \mathrm{m}$ ARC at normal incidence}

\section{Design - ZnS / YF ${ }_{3}$}

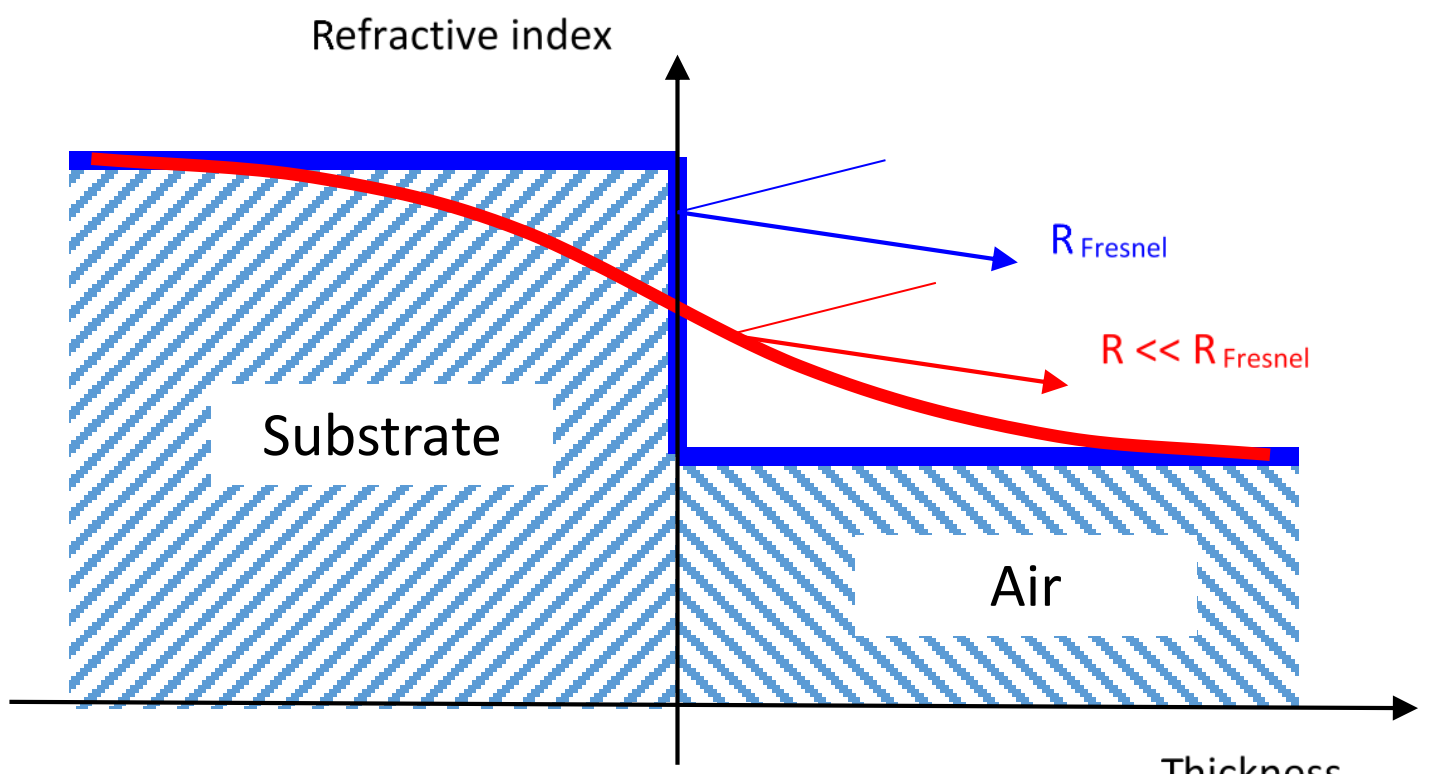

Thickness

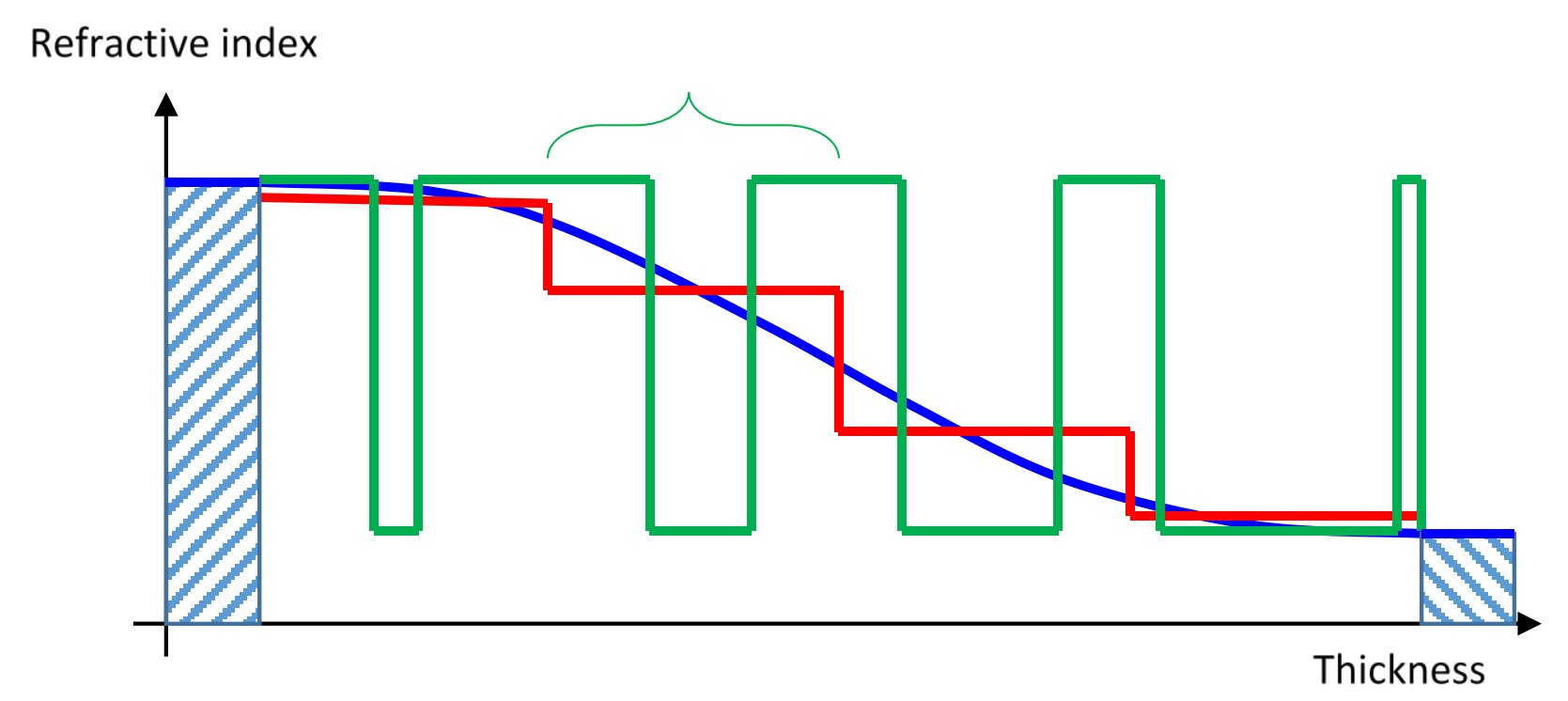




\section{5-15 $\mu \mathrm{m}$ ARC at normal incidence}

Design - ZnS $/ \mathrm{YF}_{3}$

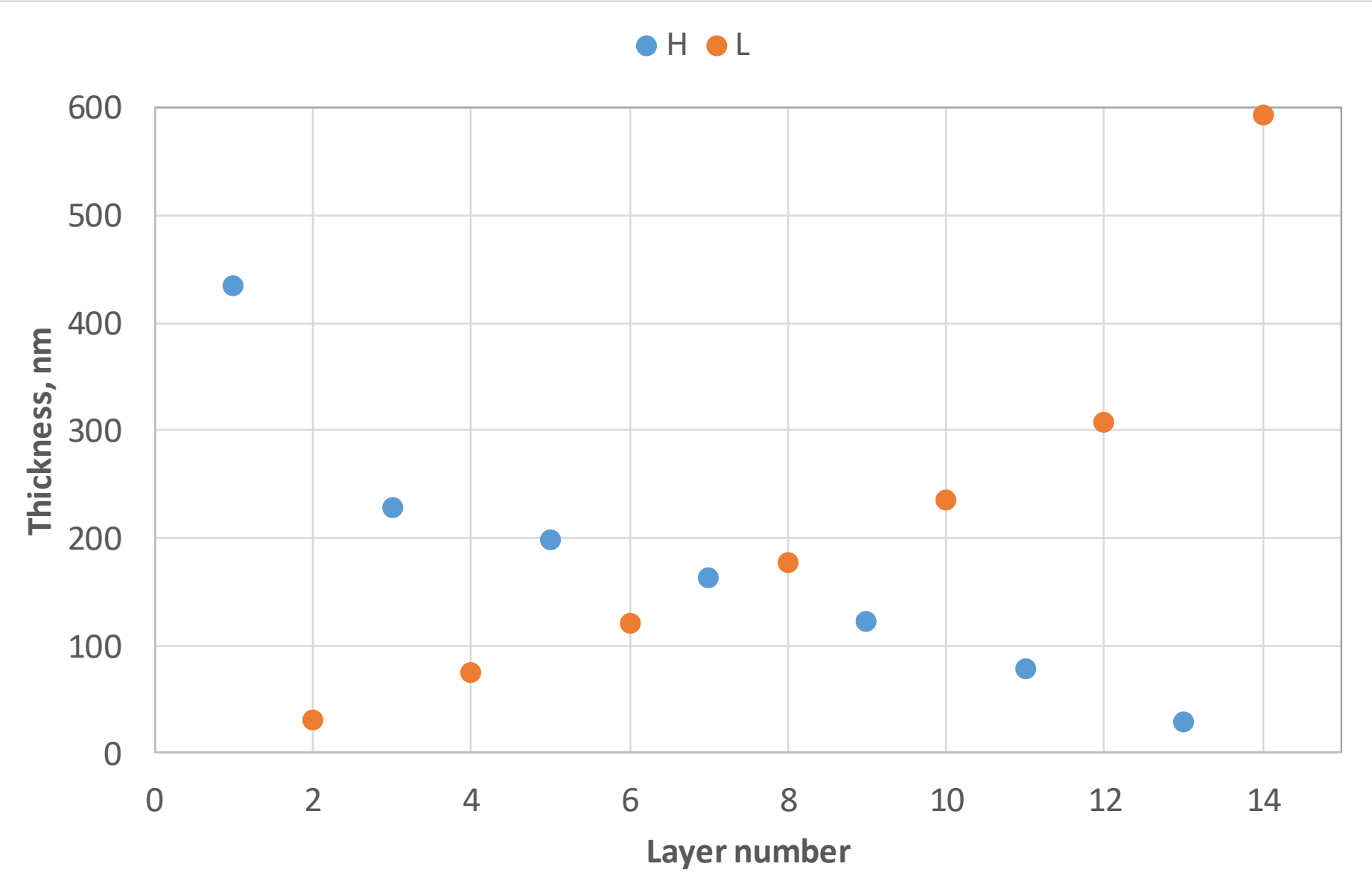

- Substrate $=\mathrm{ZnSe}$

- $\mathrm{H}=\mathrm{ZnS}$

- $\mathrm{L}=\mathrm{YF}_{3}$

- Number of layers $=14$

- Total thickness $=2800 \mathrm{~nm}$

- Thinnest layer $=30 \mathrm{~nm}$

- Thicker layer $=593 \mathrm{~nm}$ 


\section{5-15 $\mu \mathrm{m}$ ARC at normal incidence}

\section{Spectral performances}

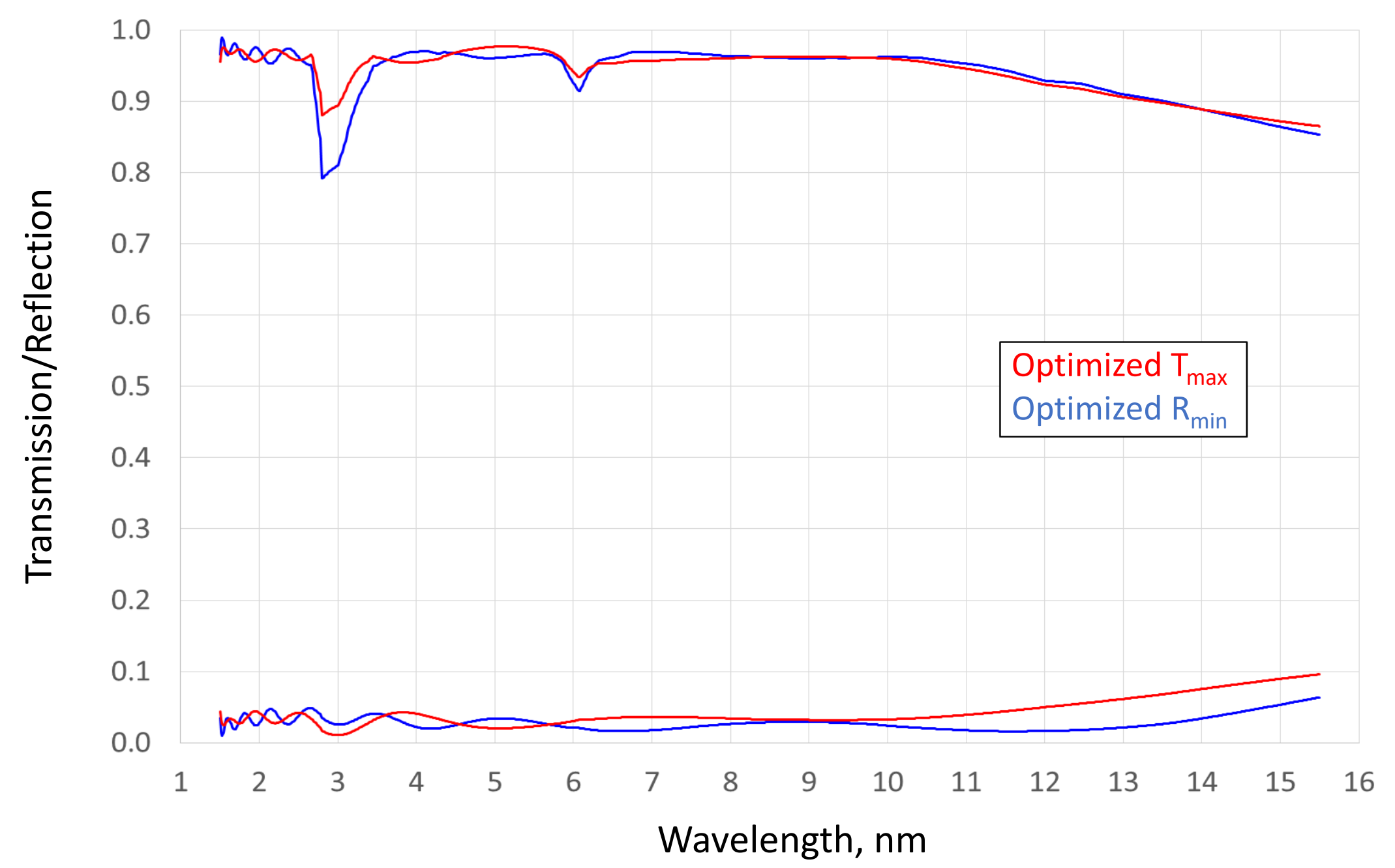




\section{5-15 $\mu \mathrm{m}$ ARC at normal incidence Bühler SYRUSpro 710}

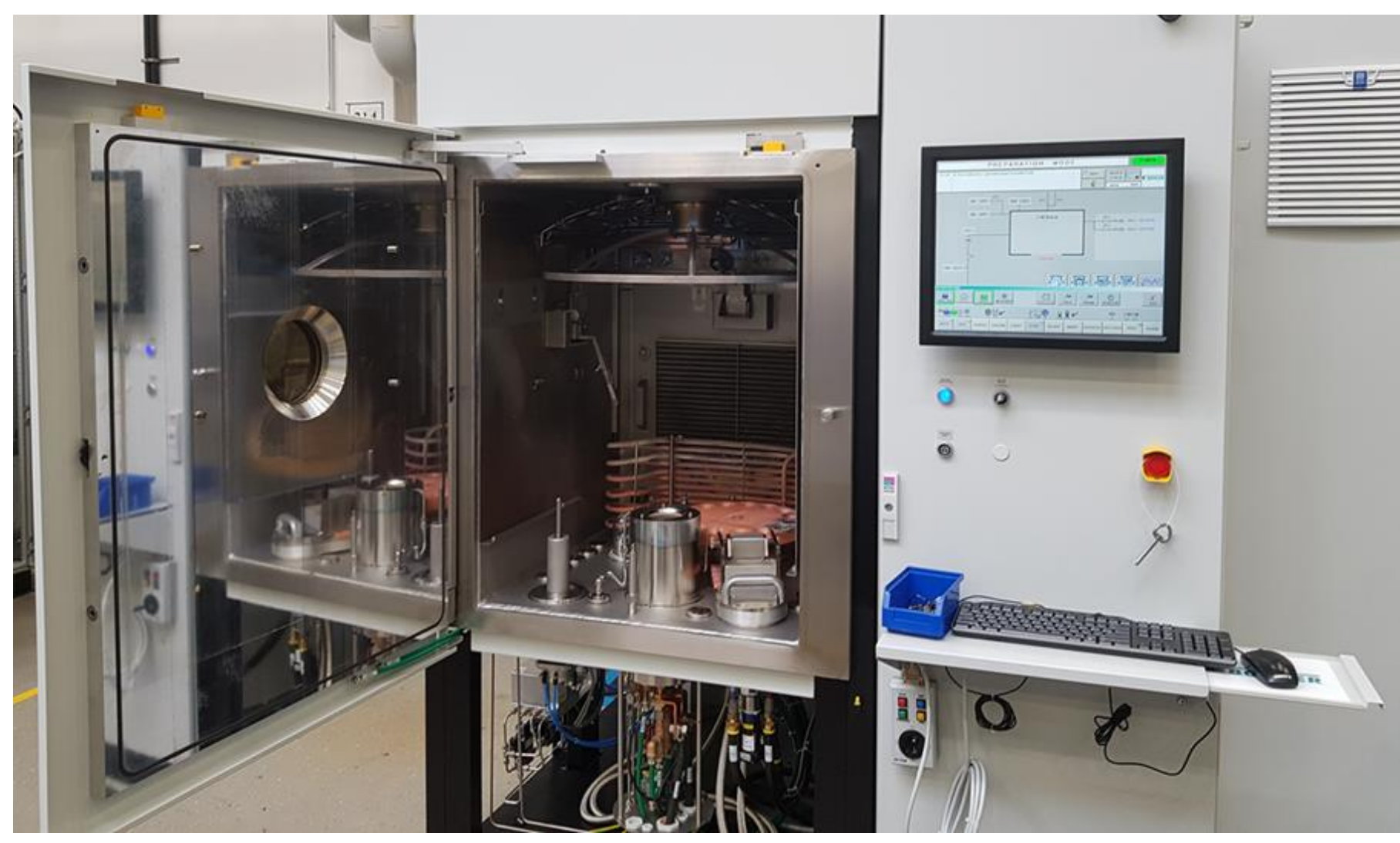

- Multiple sample holder calotte

- 2 process zones:

- 1 assistance plasma source,

- 1 e-beam

- Real time monochromatic optical monitoring of the deposition in transmission.

- Available materials: $\mathrm{ZnS}, \mathrm{YF}_{3}$, chalcogenides, metals.

- Available Gas : Ar, O2, N2. 


\section{5-15 $\mu \mathrm{m}$ ARC at normal incidence}

Experimental performances

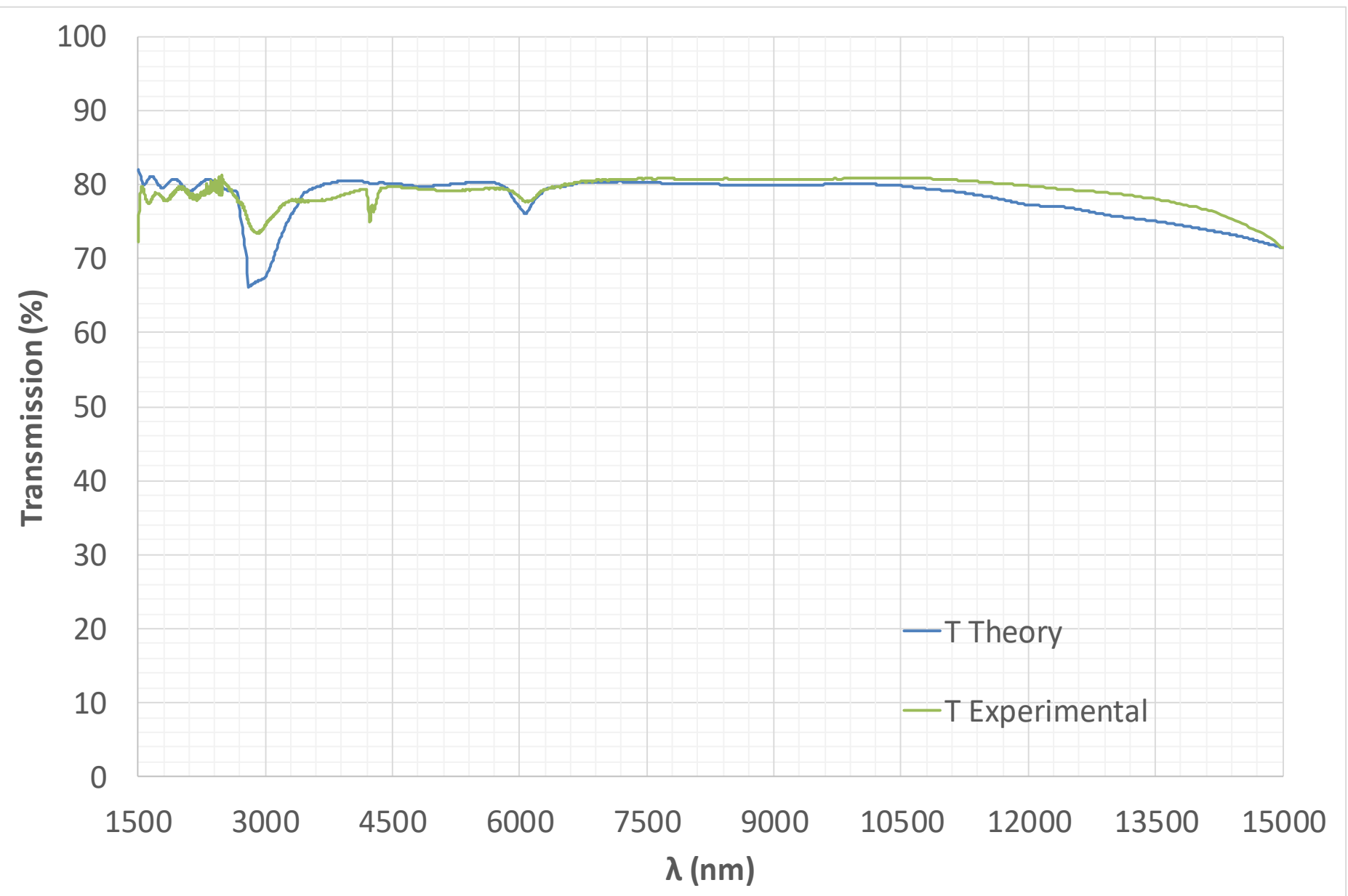




\section{Conclusions}

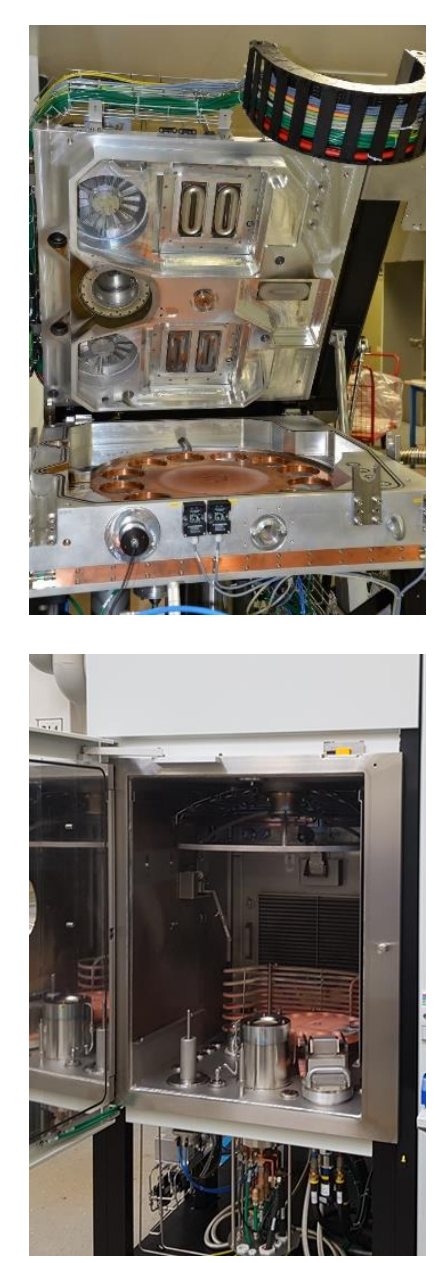

- High performances antireflection coatings can be designed with all thin-films solution.

- The performances are however limited depending on the spectral and geometrical requirements.

- Actual technology level allows fabricating all designed structures with an excellent theory/experiment agreement. 


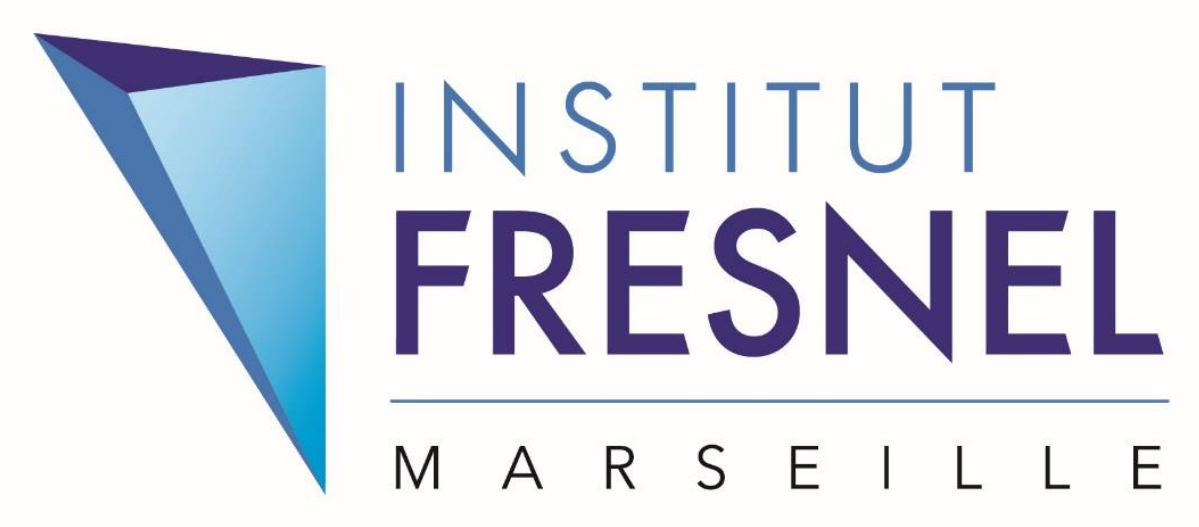

